\title{
Psychological impact of the COVID-19 pandemic on pregnant women in
}

\section{Bosnia and Herzegovina and Serbia}

Tutnjević, S. ${ }^{*}$ \& Lakić, S. ${ }^{1}$ (submitted for review). Psychological impact of the COVID-19 pandemic on pregnant women in Bosnia and Herzegovina and Serbia.

${ }^{\mathbf{1}}$ University of Banja Luka, Faculty of Philosophy, Department of Psychology.

*Corresponding author (Slavica Tutnjević): slavica.tutnjevic@ff.unibl.org 


\section{Psychological impact of the COVID-19 pandemic on pregnant women in}

\section{Bosnia and Herzegovina and Serbia}

Key words: pregnancy, COVID-19, partner support, attachment, stress, IES-R.

\section{Highlights:}

- A significant percentage of pregnant women experienced high amounts of stress during the COVID-19 pandemic

- Partner support is an important correlate of psychological response in pregnant women during the pandemic

- Secure attachment style may have a protective role in dealing with the pandemic-induced stress

- The IES-R Avoidance scale might not be a good indicator for psychological impact assessment during the pandemic

Abstract

This study investigated the psychological impact of the COVID-19 pandemic in pregnant women from Bosnia and Herzegovina and Serbia during March and April 2020.

152 respondents filled out an online administered questionnaire assessing psychological impact of the COVID-19 pandemic, fear of COVID-19 infection and death, attachment styles, perceived social support and other relevant sociodemographic and life history variables. The results indicate that the COVID-19 pandemic significantly impacts the level of fear and overall psychological functioning of the pregnant women. Especially prone to increased stress reactions are those who have lower partner support, fearful and preoccupied attachment styles, and lower financial status. The results are discussed in terms of the need for a systemic approach to psychological screening for pregnant women. We also point out 
Running head: PSYCHOLOGICAL IMPACT OF COVID-19 IN PREGNANCY

the need to carefully evaluate the use of the IES-R when applied to assess reactions to collectively experienced prolonged stressful events such as the pandemic. 
Although pregnancy is commonly believed to be a blissful time for a woman, many women become psychologically vulnerable and may experience a range of negative emotions during pregnancy. In fact, pregnancy is a major life changing event involving various hormonal and psychological changes that can increase the risk of mental health problems, particularly of depression (Bennett et al., 2004) and anxiety (Ross et al., 2006). In the "normal times", that is, before the COVID-19 pandemic, it was estimated that about $10 \%$ of pregnant women experienced a mental disorder, primarily depression (WHO, 2020), with this number being even higher (around 16\%) in developing countries.

Prolonged concern for own health and that of the loved ones, restriction of physical movement, social isolation, and the general sense of anxiety and uncertainty during the COVID-19 pandemic have all led to heightened levels of perceived stress and anxiety in the general population (Bao et al., 2020, Brooks et al., 2020, Wang et al., 2020). Furthermore, changes in the frequency and the routine of regular check-ups, lack of pregnancy-related peer-support networks, fear for the health and the life of the foetus, ban on the presence of birth support persons, and altered expectations related to perinatal and postnatal care have all been identified as important additional sources of stress for pregnant women, leading to a plausible assumption that the COVID-19-related anxiety might be particularly high in the population of pregnant women (Ali \& Feroz, 2020, Fakari \& Simbar, 2020, Matvienko-Sikar, 2020, Nanjundaswamy et al., 2020, Roy \& Pless, 2020, Thapa et al., 2020, Zeng et al., 2020).

Numerous studies have already shown that prolonged and/or severe stress in pregnancy can have serious consequences for both the mother and the baby. Maternal stress during pregnancy has been associated with negative outcomes in terms of mother's psychosocial functioning, parenting difficulties, lower infant birth weight, earlier gestational age, offspring psychopathology, alterations in brain development, and poorer socioemotional and cognitive development (Berthelot et al., 2020, Dunkel Schetter, 2011, Glover, 2011, 
Madigan et al., 2018, Tarabulsy et al., 2014). Moreover, several studies have found that the detrimental effects of exposure to high levels of prenatal maternal stress specifically related to objective natural disasters can be both pervasive and persistent in terms of the developmental outcomes for children (e.g., the Ice Storm Project, King \& Laplante, 2005, the Queensland Flood study, King et al., 2015). As the COVID-19 pandemic has exceeded the previously investigated disasters in both scope and intensity, we can presume that the psychological effects of the pandemic-related prenatal stress might be at least as detrimental, if not more.

Several empirical studies and preliminary reports dealing with the psychological consequences of the pandemic on pregnant women have been published (Berthelot et al., 2020, Corbett et al., 2020, Durankuş \& Aksu, 2020, Kotabagi et al., 2020, Lebel et al., 2020, Masjoudi et al., 2020, Milne et al., 2020, Nanjundaswamy et al., 2020, Saadati et al., 2020, Saccone et al., 2020), all pointing to the same conclusion that pregnant women experience significantly higher levels of stress and anxiety during the pandemic. The perceived levels of distress and psychiatric symptoms among pregnant women in Quebec were higher than before the pandemic, after controlling for age, gestational age, household income, education, and lifetime psychiatric disorders (Berthelot, 2020). A study in Ireland showed that over half of pregnant women $(50.7 \%)$ worried about their health often or all the time, $83.3 \%$ had heightened anxiety regarding their older relatives' health, followed by concern about their other children (66.7\%) and their unborn baby (63.4\%) (Corbett et al., 2020). A study by Kotabagi et al. in the UK (2020), showed that the pregnant women's anxiety rose to a maximum at the height of the pandemic deaths, and then decreased as the end of the pandemic seemed to approach. Saccone et al. (2020) showed that the COVID-19 outbreak had a moderate to severe psychological impact on pregnant women in Italy, with more than two-thirds of the women reporting higher than normal anxiety. Durankuş \& Aksu (2020) 
found that in Turkey, the anxiety and depression scores were significantly higher in pregnant women compared to the control group, concluding that mental disorders arise at levels two times greater than those observed under normal circumstances. Nanjundaswamy et al. (2020) performed a survey among health workers in India, 40\% of which reported having been contacted by more than ten pregnant women each because of anxiety related to COVID-19.

It seems clear from these preliminary studies that the psychological burden of the COVID-19 pandemic on pregnant women is significant, and as such imposes a threat to the general public health. Nevertheless, further studies are needed to confirm these findings in different settings, and to shed more light on different factors that may amplify or reduce prenatal stress induced by the pandemic.

The aim of our study was to investigate the psychological impact of the COVID-19 pandemic on pregnant women in Bosnia and Herzegovina and Serbia. Local authorities imposed strict lockdown rules during several weeks from March through May 2020 while the general population feared how the already impoverished and weak health system will cope with the pandemic. The lockdown measures were among the strictest in Europe, including several curfew periods from Friday afternoon through Monday morning. Because they were not thought to be a population at-risk in the beginning of the pandemic, pregnant women were left without specific instructions on how to deal with the newly arrived situation in a health system suddenly reduced to a minimal emergency-only functioning. More specifically, we sought to identify:

a) stress-induced reactions of avoidance, intrusion and hyperarousal related to the COVID-19 pandemic,

b) reported levels of fear of infection and fear of death for self, foetus, partner and close family members, 
c) factors that are known to alleviate or aggravate reactions to traumatic stress, specifically the perceived level of support by partner, mother and/or other close people (Stapleton et al., 2012, Rini et al., 2006), and attachment styles (Armour et al., 2011, Declercq \& Palmans, 2006, O'Connor \& Elklit, 2008), which become particularly relevant in pregnancy.

\section{Methods}

\section{Participants and procedure}

The data were collected online during the COVID-19 lockdown period in Bosnia and Herzegovina and Serbia from April 24 to May 31, 2020. The questionnaire was distributed via social network platforms, targeting sites intended to connect pregnant women in the region (e.g. pregnancy related Facebook pages and peer-support groups). Although they were instructed to anonymously fill out the questionnaire, the participants were left with the option to leave their email addresses if they would be willing to participate in a follow-up study. After removing data from participants who resided in countries other than Bosnia and Herzegovina or Serbia, our total sample consisted of 152 pregnant women. A large majority of them $(n=130,85.5 \%)$ were from Bosnia and Herzegovina, However, we decided to leave Serbian participants in the sample due to the similarities in the official government approaches towards the epidemic during this period and the largely shared media space covering related events. The age of the respondents ranged from 19 to $44(M=31.0, M d n=$ 31.0) years with gestation weeks ranging from 7 to $43(M=26.3, M d n=27, S D=9.1)$. Six participants $(3.9 \%)$ reported to have had complications during the current pregnancy, out of which one was hospitalized at the moment of responding to the questionnaire. None of the participants reported to have been infected with the COVID-19. 


\section{Measures}

Psychological impact of COVID-19. To assess the psychological impact we used an adapted Serbian version of the Impact of Event Scale-Revised (IES-R, Weiss \& Marmar, 1997). In the original form the IES-R is a self-administered, 22 -item questionnaire with three subscales assessing stress reactions of avodiance, intrusion, and hyperarousal. The IES-R was conceived as a self-report measure aimed to evaluate the risk for development of PTSD, particularly after a recent trauma (Hyer \& Brown, 2008). Each item is rated on a five-point scale (from 0 to 4), referring to the frequency of the symptoms. The total score for each subscale is calculated using either the sum or the arithmetic mean of the responses. For the purpose of this study, we eliminated the item 14 "I found myself acting or feeling like I was back at that time" because the pandemic was not over at the time of the our data collection, whereas this item is formulated to capture a specific event that occurred in the past. Some researchers recently used cutoff scores (e.g., Malinauskienė \& Bernotaite, 2016, Saccone et al., 2020) classifying participants into four groups dependent on the level of impact. Other researchers (Creamer, Bell, \& Failla, 2003, Rash et al. 2008) found that the average cutoff score of 1.50 provides a reasonably good predictive power for detecting PTSD. Although we were aware the author of the IES-R (Weiss, 2004) explicitly warned against the use of strict cutoff scores for diagnostic decisions, we opted to also use them for descriptive and comparison purposes. After correcting for the eliminated item, we used commensurate cut-off scores for average values to classify our participants as was done in above-mentioned studies: 0.00 to 1.08 (no impact), 1.09 to 1.49 (mild impact), 1.50 to 1.67 (moderate impact), and 1.68 and more (severe impact).

COVID-19 fear of infection and death. With a set of individual items we asked participants to separately rate their fear of becoming infected with COVID-19 and the fear of dying from the infection. Participants rated the level of how scared they were on a five-point 
scale (from "not at all" to " yes, extremely") for themselves, but also for their babies, their partners, and their close ones (parents or other close people). We conducted an exploratory factor analysis on the matrix of polychoric correlations to examine whether one can extract a latent-variable solution which would aid in analyzing our data in a more manageable way. Scree plot examination, parallel analysis and theoretical criteria suggested that both a twofactor solution with separate factors of Fear of Infection and Fear of Death, and a singlefactor solution (Fear of COVID-19) represented the common variance reasonably well. For instance, an unrotated Fear of COVID-19 factor accounted for $61 \%$ of the item common variance, with loadings ranging from .71 to .82 (minres factor extraction). On the other hand, we observed theoretically expected item loadings on Fear of Infection and Fear of Death factors with the correlation between the two amounting to .63. Internal consistency measures on all scales were high (see Table 2). Therefore, we decided to focus on the averaged scores in our analyses.

Social support during the COVID-19 outbreak. Three single-item five-point scales asked participants to rate the level of support they were receiving during the pandemic from their partners, mothers, and from significant others (parents, friends, etc.). In the analyses we also used a formative measure of a perceived social support scale by computing an average response on these items.

Attachment styles. To assess adult attachment styles we administered a globally popular self-report measure RQ (The Relationship Questionnaire; Bartholomew \& Horowitz, 1991). On this four-item questionnaire respondents evaluated the similarity of their own relationship style on a five-point scale to typical descriptions of secure, fearful, preoccupied, and dismissing style. In addition, respondents selected one style which best described them among the four. In this sample 104 (68\%) were self-classified as secure, $12(8 \%)$ as fearful, $18(12 \%)$ as preoccupied, and $18(12 \%)$ as dismissing. 
In addition to the above the questionnaire addressed a range of socio-demographic questions (age, gestation week, level of education, financial status, number of children), as well as relevant life history variables (history of miscarriage, history of seeked psychological treatment, increased-risk pregnancy).

\section{Data analysis}

After we verified the data for the validity of responses we conducted the descriptive analysis for all single-item and multi-item scales. Internal consistency coefficients were computed and reported for the multi-item reflective scales (IES-R and COVID-19 Fear). We performed exploratory factor analysis (minres method, oblimin rotation) using a matrix of polychoric correlation to determine the structure of responses to the COVID-19 Fear items, since this was a measure constructed for the purposes of the study. To present the associations among the variables we chose to report correlation coefficients which take into account the level of measurement of the variables at hand (e.g. in case one variable was dimensional and the other categorical ordinal we reported polyserial coefficients). Furthermore, since our sample was relatively small, with some variables being heavily tailed and thus, estimates susceptible to the influence of outliers and distribution effects, we reported rank-based coefficients whenever a dimensional variable was involved in an analysis (see de Winter, Gosling, and Potter, 2016 for the arguments). Similarly, we performed both average-score and rank-score analysis when testing for differences between the groups, and when conclusions were inconsistent we reported all results. For inferential purposes we calculated Bayes factors with weakly informative priors (stretched Beta prior with 0.5 parameter for correlations and Cauchy prior with 0.707 parameter for testing differences between two groups) having in mind that our research was exploratory to a large extent (see Wagenmakers et al., 2018). To graphically depict the model of all variables that were 
empirically associated with the criteria, we conducted a network analysis on a matrix of relevant correlation coefficients. All analyses were conducted using JASP (JASP Team, 2020) and R 4.0.1 (R Core Team, 2020) with packages polycor (Fox, 2019), psych (Revelle, 2019), and qgraph (Epskamp et al., 2012).

\section{Results}

Although we did not have a direct comparison group, the very distribution of the IESR scales' scores (Table 1) suggests that the early phase of the pandemic had a noticeable psychological impact on pregnant women in our sample. By using the proposed cutoff scores (analogous to Saccone et al., 2020) we see that 52 respondents $(34.2 \%)$ experienced a severe psychological impact, additional 15 (9.9\%) experienced moderate, $35(23.0 \%)$ mild impact. Approximately one-third of the respondents $(n=50,32.9 \%)$ were in the range considered to reflect normal reactions to stressful conditions. By analyzing the contribution of individual IES-R components, it becomes obvious that avoidance reactions accounted for the largest amount of high values, followed by intrusion and then hyperarousal reactions. While the median response on the avoidance items was "moderately" with the whole distribution being visibly negatively asymmetrical, the average response on the hyperarousal scale was "rarely" with the heavily positive distribution, meaning that a majority of respondents had low scores.

\section{INSERT TABLE 1 HERE}

Table 2 demonstrates the effects of the COVID-19 epidemic on pregnant women from another, more direct perspective. Specifically, slightly more than one-third of the respondents reported to have felt moderate to intense fear of infection for themselves or their babies, 
whereas slightly more than $50 \%$ of the respondents reported to have felt so for their partners or other close persons. Naturally, the fear of death from COVID-19 was less pronounced, but still, every third respondent felt moderate, considerable, or intense fear for close others, while every fifth respondent reported to have feared for the life of the baby to such extent.

\section{INSERT TABLE 2 HERE}

The data presented in Table 3 lead to several conclusions regarding the measures used as dependent variables. First, with regard to the structure of the IES-R, the reactions of intrusion and hyperarousal correlate more strongly with each other than they do with avoidant reactions - where correlations could be described as only moderate from a purely statistical standpoint. This finding also explains significantly larger correlations of intrusion and hyperarousal scales with the total IES-R score. Second, logically assumed relations between the fear and psychological impact were confirmed, but simultaneously we observed only a small correlation between the IES-R avoidance and the fear scales. Moreover, when this association was statistically controlled via linear regression analysis for the combination of variables intrusion and hyperarousal, it completely disappeared (e.g. for the Fear of COVID19 scale as dependent variable: before controlling $\beta=0.17, \mathrm{p}=.056$; after controlling $\beta=$ $0.03, \mathrm{p}=.741, \mathrm{sr} 2<.001)$. In other words, our data suggest that there is a much larger share of the variance in avoidance scores not stemming directly from the fear affect.

\section{INSERT TABLE 3 HERE}

Partner support and financial status were the only variables which consistently correlated with the COVID-19 related fear and reactions potentially leading to posttraumatic 
stress (Table 4). On the other hand, attachment styles correlated noticeably only with the IESR scales. More pronounced secure attachment style appears to be a protective factor, as opposed to fearful and preoccupied attachment styles, which seem to aggravate the psychological impact of COVID-19 related stress. We found no apparent correlations between dismissing attachment style and reactions to the stressful event. When the data were analyzed using self-classified attachment types we observed similar findings with the following pattern of scores being obtained for virtually all scales: fearful > preoccupied $>$ secure $>$ dismissing. The largest differences were observed for hyperarousal and intrusion scales, but due to small subsample sizes, and consequently, low statistical power, the levels of nominal statistical significance were reached only for the classical analysis of variance (Hyperarousal: $\eta 2=.07$, ANOVA $p=.011$, Welch corrected $p=.093$, Kruskal-Wallis $p=$ .069 ; Intrusion: $\eta 2=.05$, ANOVA $\mathrm{p}=.046$, Welch corrected $\mathrm{p}=.152$, Kruskal-Wallis $\mathrm{p}=$ .093). The correlations between fear of COVID-19 and perceived support by the mother and significant others were low and relatively contradictory. In addition, we found a suggestive correlation between perceived support from the mother and avoidance reactions. Interestingly, we did not observe any indicative associations of perceived stress and fear with age and gestation week, even after graphically investigating possible nonlinear relations which could have not been signalled by correlation coefficients.

\section{INSERT TABLE 4 HERE}

We also tested for the effects of several control variables that we believed might have increased the susceptibility to the impact of this stressful event. We found substantively suggestive, but statistically inconclusive effects of increased-risk pregnancies (e.g. Fear of 
COVID-19: $\mathrm{t}(57.1)=2.16, \mathrm{p}=.036, \mathrm{~d}=0.48, \mathrm{BF} 10=3.74$; IES-R Intrusion: $\mathrm{t}(51.06)=1.51$, $\mathrm{p}=.135, \mathrm{~d}=0.29, \mathrm{BF} 01=1.75)$, and a history of treatment for psychological problems (e.g. Fear of death: $\mathrm{t}(40.2)=2.07, \mathrm{p}=.044, \mathrm{~d}=0.51, \mathrm{BF} 10=4.03$; IES-R Hyperarousal: $\mathrm{t}(42.9)=$ $1.50, \mathrm{p}=.142, \mathrm{~d}=0.34, \mathrm{BF} 01=1.27)$. A history of miscarriages had a noticeable association only with avoiding dealing with COVID-19 topics $(\mathrm{t}(54.5)=2.38, \mathrm{p}=.021, \mathrm{~d}=0.48, \mathrm{BF} 10=$ 3.18; for other scales $d<.16$ ). We found no plausible indication that having other children plays a significant role per se in increasing stress reactions of pregnant women to the COVID-19 epidemic (all ds $<.20$, all ps $>.260$, BF01 ranging from 3.0 to 5.6). It is important to emphasize that significant findings on the effects of partner support, financial status, and attachment styles held - or even got more pronounced - after we discarded the respondents with current increased-risk pregnancies, the history of miscarriage and the history of psychological treatment - using the sample of 78 respondents.

Since there were significant associations among the correlates, we wanted to produce a descriptive model which would take into account the multivariate nature of the phenomenon. Figure 1 presents a network model of interrelationships among five criterion variables and ten aforementioned correlates. For instance, one can more easily notice the distinctive characteristics of the avoidance variable within this context. In contrast to other criterion variables, avoidance had a non-central position within the model, without being associated with the variables such as partner support, attachment styles, increased-risk pregnancy or the history of psychological treatment.

\section{INSERT FIGURE 1 HERE}




\section{Discussion}

Our study provides empirical evidence that the COVID-19 pandemic significantly impacts the psychological health of many pregnant women. Such results were expected in light of both the severity of the crisis caused by the pandemic, and the psychological vulnerability of pregnant women in general. They are also in line with the findings of other authors who identified higher levels of stress in pregnant women across the world (Berthelot et al., 2020, Corbett et al., 2020, Durankuş \& Aksu, 2020, Kotabagi et al., 2020, Lebel et al., 2020, Masjoudi et al., 2020, Nanjundaswamy et al., 2020, Saadati et al., 2020, Saccone et al., 2020). Our study suggests that the intensity of fear of COVID-19 directly affects the stress response, but that there are also several psychological factors modulating this relationship.

By using the approach of Creamer et al. (2003) and Rash et al. (2008), who - based on empirical studies - suggested that the overall cutoff score of 1.5 is a reasonable measure for detecting risk of developing PTSD, we find that $44.1 \%$ of our respondents would be at such risk. This is less than what was found by Saccone et al. (2020) in Italy, where as much as $61 \%$ had such a IES-R score (falling into a moderate or severe group). This difference could be explained by the fact that Italy was among the worst affected countries in the world at the time of data collection (March 15 to April 1, 2020), while Bosnia and Herzegovina and Serbia had a small number of cases and only a handful of fatalities at the time when we collected the data. Still, this percentage is quite high having in mind long-term consequences of enduring stress on pregnant women and their babies.

Nevertheless, it is reasonable to ask whether the total IES-R score should be regarded as a valid measure of adverse reactions to stress when it comes to the COVID-19 pandemic. Namely, one of its subscales - avoidance - seems to be capturing reactions that correlate only weakly or negligibly with COVID-19 related fears and other relevant variables from our final 
model (partner support, attachment styles, increased-risk pregnancy or the history of psychological treatment). This subscale includes the statements such as: "I tried not to talk about it.", "I tried not to think about it." More than two thirds of our participants (68.4\%) reported having tried to avoid thinking about COVID-19-related content sometimes or more often. While these statements do point to the importance and omnipresence of the traumatic event of the pandemic in everyday life of the pregnant women, they do not necessarily mean that their reactions to stress were excessive or pathological in any way. Rather, in our view, they might represent typical adaptive reactions to the information overload related to COVID-19 that permeated every aspect of living during the first months of the pandemic.

On the other hand, the hyperarousal scale, consisting of statements such as: "Reminders of it caused me to have physical reactions, such as sweating, trouble breathing, nausea, or a pounding heart." and "I felt watchful and on-guard.", and the intrusion scale, consisting of statements such as "Any reminder brought back feelings about it." and "Pictures about it popped into my mind." might actually point to some more intense and persistent effects of the traumatic event in the daily life of these women. Yet, as can be seen from the results, the scores were considerably lower on the intrusion scale, with $38.2 \%$ of the women experiencing intrusive thoughts and feelings on average sometimes or more often. Finally, $25.7 \%$ of our respondents experienced hyperarousal symptoms on average sometimes or more often, while $6.6 . \%$ reported having felt hyperarousal symptoms frequently or all the time. In summary, the overall score on the Revised Impact of Event Scale (IES-R) might be less informative than the specific reactions measured by the individual subscales. Other researchers and practitioners should probably focus more on the hyperarousal and intrusion indicators when assessing stress related to collectively experienced traumatic events of prolonged duration such as the COVID-19 pandemic (e.g. see Hyer \& Brown, 2008, for differences in predictive values of the subscales). 
Approximately every third pregnant woman was moderately to intensely scared to become themselves infected with COVID-19, while approximately every other pregnant woman felt so for their partner and significant others. In comparison to that, the fear of death was relatively low, with $8 \%$ of the respondents being moderately to intensely scared for their own life and $18 \%$ for their babies. The low percentage of women reporting "considerable" or "intense" fear of death for themselves or their babies might also reflect the official guidelines in the first months of the pandemic according to which pregnant women and their babies were not considered at-risk population in terms of the COVID-19 (Qiao, 2000). In the meantime, the latest evidence has suggested that pregnant women might be at increased risk for severe COVID-19 illness in comparison to non-pregnant women (Ellington et al., 2020), which might further exacerbate the anxiety in this population in the future, all the more so since media reports were published confirming deaths of pregnant women infected with COVID-19 in July 2020 in Serbia. As expected, knowing that the vulnerability to COVID-19 increases with age, we observed that the fear of death was highest for significant others, most probably, the parents. Approximately $15 \%$ of the respondents reported "considerable" or "intense" fear which in itself is a source of considerable prenatal stress.

Not surprisingly, our findings confirm that perceived partner support plays an important role in the mental health of pregnant women, as known from previous studies (Stapleton et al., 2012, Rini et al., 2006). Severity of the psychological impact of the pandemic and the fear of death were more pronounced in women who perceived the partner support as low. Surprisingly, we found no evidence that support from the mothers plays a protective role (we even observed correlations in the opposite direction) - and neither does the support from significant others. Furthermore, our results suggest that although attachment security does not correlate with the intensity of the COVID-19 related fear, it may act as a protective factor against the severity of its psychological impact. Indeed, previous studies 
showed that secure attachment correlates negatively with the development of PTSD symptoms (Declercq \& Palmans, 2006, O'Connor \& Elklit, 2008), depression and anxiety in response to trauma (Armour et al., 2011). We observed a positive correlation between the psychological impact of the pandemic and the fearful and preoccupied attachment styles, respectively, which is in line with the above-mentioned previous studies. This correlation is stronger for the hyperarousal and intrusion than for the avoidance. As already pointed out, avoiding to think or talk about the pandemic in this context should not necessarily be considered as an dysfunctional reaction to stress. On the other hand, the dismissing attachment style did not correlate with the severity of the psychological impact of the pandemic.

The main limitation of our study is a relatively small sample collected online on a voluntary basis. As such, it is not representative of the general population of pregnant women. In fact, our sample consisted mostly of educated women, regular users of the internet and social networks. In addition, pregnant women from Bosnia and Herzegovina and Serbia represent a special subsample inevitably affected by social, economical and cultural factors characteristic of these two countries at the specific period of the first two months of the pandemic, and as such should be taken with caution when talking about pregnant women in general. Another important limitation is the fact that we had no comparison sample of preCOVID-19 pregnant women, so we had to draw our conclusions based on the descriptive data and cutoff scores used in similar studies. Nevertheless, our results are in line with those found in similar studies which did have a comparison sample (e.g. Durankuş \& Aksu, 2020, Lebel et al., 2020), showing that pregnant women are experiencing substantial psychological distress whose levels far exceed those normally expected during pregnancy and those experienced by other groups of people during the pandemic. Finally, while we did try to identify factors that might amplify or reduce reactions to stress of our respondents, no causal 
inferences regarding the influence of attachment styles and partner support on the psychological impact of the pandemic are possible. A larger picture of individual histories, risk and protective factors which might be at interplay in every individual woman cannot be accounted for by the factors emphasized in our study alone.

\section{Conclusions}

Despite the limitations, our study clearly underlines the importance of psychological support for pregnant women during the COVID-19 pandemic. The enduring feelings of fear, anxiety, and uncertainty, if untreated, might have long-term consequences on the mental health of the mothers but also on the physical and psychological development of the babies. This is particularly important for pregnant women with histories of miscarriage and psychological treatment, those with increased-risk pregnancies and those in challenging financial situations. Our findings suggest that throughout the duration of the pandemic, perceived partner support and attachment styles should be an important part of the regular psychological screening for pregnant women, with low partner support and fearful and preoccupied attachment styles serving as indicators of the need for continuous psychological support. This approach might be helpful in preventing negative consequences of the pandemic on the psychological health of the mothers and the developmental outcomes of the babies. 


\section{References}

Ali, N. A., \& Feroz, A. (2020). Maternal mental health amidst the COVID-19 pandemic. Asian Journal of Psychiatry, (54), 102261.

Armour, C., Elklit, A., \& Shevlin, M. (2011). Attachment typologies and posttraumatic stress disorder (PTSD), depression and anxiety: a latent profile analysis approach. European Journal of Psychotraumatology, 2(1), 6018.

Bao, Y., Sun, Y., Meng, S., Shi, J., \& Lu, L. (2020). 2019-nCoV epidemic: address mental health care to empower society. The Lancet, 395(10224), e37-e38.

Bartholomew, K. \& Horowitz, L. M. (1991). Attachment styles among young adults: A test of a four-category model. Journal of Personality and Social Psychology, 61, 226-244.

Bennett, H. A., Einarson, A., Taddio, A., Koren, G., \& Einarson, T. R. (2004). Prevalence of depression during pregnancy: systematic review. Obstetrics \& Gynecology, 103(4), 698-709.

Berthelot, N., Lemieux, R., Garon Bissonnette, J., Drouin Maziade, C., Martel, É., \& Maziade, M. (2020). Uptrend in distress and psychiatric symptomatology in pregnant women during the COVID 19 pandemic. Acta Obstetricia et Gynecologica Scandinavica.

Brooks, S. K., Webster, R. K., Smith, L. E., Woodland, L., Wessely, S., Greenberg, N., \& Rubin, G. J. (2020). The psychological impact of quarantine and how to reduce it: rapid review of the evidence. The Lancet, 395, 912-920.

Buekens P, Alger J, Breart G, Cafferata ML, Harville E, Tomasso G. (2020). A call for action for COVID-19 surveillance and research during pregnancy. Lancet Global Health, S2214-109X(20)30206-0. Online ahead of print 
Corbett, G. A., Milne, S. J., Hehir, M. P., Lindow, S. W., \& O'connell, M. P. (2020). Health anxiety and behavioural changes of pregnant women during the COVID19 pandemic. European Journal of Obstetrics, Gynecology, and Reproductive Biology, 249, 96-97.

Creamer, M., Bell, R., \& Failla, S. (2003). Psychometric properties of the impact of event scale—revised. Behaviour research and therapy, 41(12), 1489-1496.

Declercq, F., \& Palmans, V. (2006). Two subjective factors as moderators between critical incidents and the occurrence of post traumatic stress disorders: Adult attachment and perception of social support. Psychology and Psychotherapy: Theory, Research and Practice, 79(3), 323-337.

Dunkel Schetter, C. (2011). Psychological science on pregnancy: stress processes, biopsychosocial models, and emerging research issues. Annual review of psychology, 62, 531-558.

Durankuş, F., \& Aksu, E. (2020). Effects of the COVID-19 pandemic on anxiety and depressive symptoms in pregnant women: a preliminary study. The Journal of Maternal-Fetal \& Neonatal Medicine, 1-7.

Epskamp, S. Cramer, A., Waldorp, L., Schmittmann, V. \& Borsboom, D. (2012). qgraph: Network Visualizations of Relationships in Psychometric Data. Journal of Statistical Software, 48(4), 1-18. URL http://www.jstatsoft.org/v48/i04/.

Fakari, F. R., \& Simbar, M. (2020). Coronavirus Pandemic and Worries during Pregnancy; a Letter to Editor. Archives of Academic Emergency Medicine, 8(1).

Fox, J. (2019). polycor: Polychoric and Polyserial Correlations. R package version 0.710. https://CRAN.R-project.org/package=polycor Ellington, S., Strid, P., Tong, VT., et al. (2020). Characteristics of Women of Reproductive Age with Laboratory-Confirmed SARS-CoV-2 Infection by 
Pregnancy Status - United States, January 22-June 7, 2020. MMWR Morbidity and Mortality Weekly Report, 69, 769-775.

Glover V. (2011). Annual research review: prenatal stress and the origins of psychopathology: an evolutionary perspective. Journal of Child Psychology and Psychiatry, 2, 356-367.

Horowitz, M. Wilner, N. \& Alvarez, W. (1979). Impact of Event Scale: A measure of subjective

stress. Psychosomatic Medicine, 41, 209-218.

Hyer, K., \& Brown, L. M. (2008). The Impact of Event Scale-Revised: A quick measure of a patient's response to trauma. AJN The American Journal of Nursing, 108(11), 60-68.

King, S., Kildea, S., Austin, M. P., Brunet, A., Cobham, V. E., Dawson, P. A., ... \& McIntyre, H. D. (2015). QF2011: a protocol to study the effects of the Queensland flood on pregnant women, their pregnancies, and their children's early development. BMC Pregnancy \& Childbirth, 15(1), 109.

JASP Team (2020). JASP (Version 0.12) [Computer software].

King, S., \& Laplante, D. P. (2005). The effects of prenatal maternal stress on children's cognitive development: Project Ice Storm. Stress, 8(1), 35-45.

Kotabagi, P., Fortune, L., Essien, S., Nauta, M., \& Yoong, W. (2020). Anxiety and depression levels among pregnant women with COVID 19. Acta Obstetricia et Gynecologica Scandinavica. 99(7), 953-954.

Lebel, C., MacKinnon, A., Bagshawe, M., Tomfohr-Madsen, L., \& Giesbrecht, G. (2020). Elevated depression and anxiety among pregnant individuals during the COVID-19 pandemic. (preprint) 
Madigan, S., Oatley, H., Racine, N., Fearon, R. P., Schumacher, L., Akbari, E., ... \& Tarabulsy, G. M. (2018). A meta-analysis of maternal prenatal depression and anxiety on child socioemotional development. Journal of the American Academy of Child \& Adolescent Psychiatry, 57(9), 645-657.

Malinauskienè, V., \& Bernotaite, L. (2016). The Impact of Event Scale-Revised: psychometric properties of the Lithuanian version in a sample of employees exposed to workplace bullying. Acta medica Lituanica, 23(3), 185.

Masjoudi, M., Aslani, A., Khazaeian, S., \& Fathnezhad-Kazemi, A. (2020). Explaining the experience of prenatal care and investigating the association between psychological factors with self-care in pregnant women during COVID-19 pandemic: a mixed method study protocol. Reproductive health, 17(1), 1-7.

Matvienko-Sikar K, Meedya S RC. Perinatal Mental Health during the COVID-19 Pandemic. Women and Birth. British Journal of General Practice.

Milne, S. J., Corbett, G. A., Hehir, M. P., Lindow, S. W., Mohan, S., Reagu, S., ... \& O'Connell, M. P. (2020). Effects of isolation on mood and relationships in pregnant women during the covid-19 pandemic. European Journal of Obstetrics and Gynecology and Reproductive Biology.

Nanjundaswamy, M. H., Shiva, L., Desai, G., Ganjekar, S., Kishore, T., Ram, U., ... \& Chandra, P. S. (2020). COVID-19 Related Anxiety and Concerns Expressed by Pregnant and Postpartum Women-a Survey Among Obstetricians. Retrieved 20 July 2020 from https://assets.researchsquare.com/files/rs-38004/v1/c7d8d894-

\section{d19d-4845-87dd-baf775ce9fdd.pdf}

O'Connor, M., \& Elklit, A. (2008). Attachment styles, traumatic events, and PTSD: A cross-sectional investigation of adult attachment and trauma. Attachment \& human development, 10(1), 59-71. 
Qiao, J. (2020). What are the risks of COVID-19 infection in pregnant women? The Lancet, 395(10226), 760-762.

R Core Team (2020). R: A language and environment for statistical computing. $\mathrm{R}$ Foundation for Statistical Computing, Vienna, Austria. URL https://www.Rproject.org/.

Rash, C. J., Coffey, S. F., Baschnagel, J. S., Drobes, D. J., \& Saladin, M. E. (2008). Psychometric properties of the IES-R in traumatized substance dependent individuals with and without PTSD. Addictive behaviors, 33(8), 1039-1047.

Revelle, W. (2019). psych: Procedures for Personality and Psychological Research. https://CRAN.R-project.org/package=psych Version $=1.9 .12$.

Rini, C., Schetter, C. D., Hobel, C. J., Glynn, L. M., \& Sandman, C. A. (2006). Effective social support: Antecedents and consequences of partner support during pregnancy. Personal Relationships, 13(2), 207-229.

Ross, L. E., \& McLean, L. M. (2006). Anxiety Disorders During Pregnancy and the Postpartum Period: A Systematic Review. The Journal of Clinical Psychiatry, 67(8), 1285-1298. https://doi.org/10.4088/JCP.v67n0818

Roy, A. S., \& Ples, D. (2020). The impact of COVID-19 on identifying and managing perinatal anxiety. British Journal of General Practice.

Saadati, N., Afshari, P., Boostani, H., Beheshtinasab, M., Abedi, P., \& Maraghi, E. (2020). Health Anxiety of Pregnant Women and Its Related Factors During the Pandemic of Corona Virus. Retrieved 27 July 2020 from https://assets.researchsquare.com/files/rs-26840/v1/ca5edddb-b342-43d2-938d71cd234e131f.pdf

Saccone, G., Florio, A., Aiello, F., Venturella, R., De Angelis, M. C., Locci, M., ... \& Sardo, A. D. S. (2020). Psychological impact of coronavirus disease 2019 in 
pregnant women (Research letter). American Journal of Obstetrics \& Gynecology.

Stapleton, L. R. T., Schetter, C. D., Westling, E., Rini, C., Glynn, L. M., Hobel, C. J., \& Sandman, C. A. (2012). Perceived partner support in pregnancy predicts lower maternal and infant distress. Journal of family psychology, 26(3), 453-463.

Thapa, S. B., Mainali, A., Schwank, S. E., \& Acharya, G. (2020). Maternal mental health in the time of the COVID 19 pandemic. Acta Obstetricia et Gynecologica Scandinavica, 99, 817-818.

Tarabulsy, G. M., Pearson, J., Vaillancourt-Morel, M. P., Bussières, E. L., Madigan, S., Lemelin, J. P., ... \& Royer, F. (2014). Meta-analytic findings of the relation between maternal prenatal stress and anxiety and child cognitive outcome. Journal of Developmental \& Behavioral Pediatrics, 35(1), 38-43.

Wagenmakers, E. J., Love, J., Marsman, M., Jamil, T., Ly, A., Verhagen, J., ... \& Meerhoff, F. (2018). Bayesian inference for psychology. Part II: Example applications with JASP. Psychonomic Bulletin \& Review, 25(1), 58-76.

Wang, C., Pan, R., Wan, X., Tan, Y., Xu, L., Ho, C. S., \& Ho, R. C. (2020). Immediate psychological responses and associated factors during the initial stage of the 2019 coronavirus disease (COVID-19) epidemic among the general population in China. International journal of environmental research and public health, 17(5), 1729.

Weiss, D.S. (2004). The Impact of Event Scale - Revised. In J.P. Wilson, \& T.M. Keane (Eds.), Assessing psychological trauma and PTSD, (pp. 168-189). New York: Guilford press. 
Weiss, D.S., \& Marmar, C.R. (1997). The Impact of Event Scale-Revised. In J.P. Wilson \& T.M. Keane (Eds.), Assessing Psychological Trauma and PTSD, (pp.399-411). New York: Guilford press.

World Health Organization. (2020). Maternal health. Retrieved 20 July 2020 from https://www.who.int/mental_health/maternal-child/maternal_mental_health/en/

Zeng, L. N., Chen, L. G., Yang, C. M., Zeng, L. P., Zhang, L. Y., \& Peng, T. M. (2020). Mental health care for pregnant women in the COVID-19 outbreak is urgently needed. Women and Birth (in press). 
Running head: PSYCHOLOGICAL IMPACT OF COVID-19 IN PREGNANCY

Table 1

IES-R Descriptive Statistics

\begin{tabular}{lcccccccc}
\hline IES-R Scale & $M(S D)$ & Mdn $(M A D)$ & Min & Max & Sk & Mild & Moderate & Severe \\
& & & & & & & \\
\hline Total & $1.41(0.70)$ & $1.33(0.71)$ & 0 & 3 & 0.17 & $23.0 \%$ & $9.9 \%$ & $34.2 \%$ \\
Intrusion & $1.31(0.87)$ & $1.29(1.06)$ & 0 & 3.57 & 0.32 & $18.4 \%$ & $3.3 \%$ & $34.9 \%$ \\
Avodiance & $1.82(0.77)$ & $2.00(0.74)$ & 0 & 3.38 & -0.48 & $14.5 \%$ & $5.9 \%$ & $62.5 \%$ \\
& & & & & & & & \\
Hyperarousal & $0.97(0.86)$ & $0.75(0.86)$ & 0 & 3.67 & 0.88 & $9.2 \%$ & $4.6 \%$ & $21.1 \%$ \\
\end{tabular}

Note. $\mathrm{MAD}=$ Mean absolute deviation; $\mathrm{Sk}=$ Skewness. 
Running head: PSYCHOLOGICAL IMPACT OF COVID-19 IN PREGNANCY

Table 2

Fear of COVID-19 Descriptive Statistics

\begin{tabular}{|c|c|c|c|c|c|c|c|c|}
\hline Fear of COVID-19 & $M(S D)$ & $M d n$ & Min & $\operatorname{Max}$ & $S k$ & Moderate & Considerable & Intense \\
\hline Infection & $1.48(0.97)$ & 1.25 & 0 & 4 & 0.54 & & & \\
\hline Participant & $1.26(1.01)$ & 1.16 & 0 & 4 & 0.64 & $27.0 \%$ & $6.6 \%$ & $3.3 \%$ \\
\hline Baby & $1.26(1.12)$ & 1.11 & 0 & 4 & 0.65 & $23.7 \%$ & $9.2 \%$ & $4.6 \%$ \\
\hline Partner & $1.64(1.12)$ & 1.52 & 0 & 4 & 0.43 & $30.3 \%$ & $12.5 \%$ & $7.9 \%$ \\
\hline Others & $1.76(1.13)$ & 1.63 & 0 & 4 & 0.39 & $29.6 \%$ & $15.1 \%$ & $9.2 \%$ \\
\hline Death & $0.72(0.87)$ & 0.38 & 0 & 4 & 1.53 & & & \\
\hline Participant & $0.40(0.82)$ & 0.17 & 0 & 4 & 2.33 & $5.9 \%$ & $2.6 \%$ & $1.3 \%$ \\
\hline Baby & $0.69(1.00)$ & 0.34 & 0 & 4 & 1.42 & $11.8 \%$ & $5.3 \%$ & $2.0 \%$ \\
\hline Partner & $0.64(0.97)$ & 0.32 & 0 & 4 & 1.51 & $9.2 \%$ & $5.9 \%$ & $1.3 \%$ \\
\hline Others & $1.14(1.17)$ & 0.91 & 0 & 4 & 0.75 & $17.1 \%$ & $11.8 \%$ & $3.9 \%$ \\
\hline Overall & $1.10(0.84)$ & 0.98 & 0 & 4 & 1.00 & & & \\
\hline
\end{tabular}

Note. $M d n=$ Interpolated median for single-item ordinal scales; $S k=$ Skewness. Frequency of responses for options "None" and "Mild" are not shown. 
Running head: PSYCHOLOGICAL IMPACT OF COVID-19 IN PREGNANCY

Table 3

Intercorrelations among IES-R and COVID-19 Fear Scales

\begin{tabular}{lcccccc}
\hline Variable & 2 & 3 & 4 & 5 & 6 & 7 \\
& & & & & & \\
\hline 1. IES-R Total & .91 & .74 & .89 & .34 & .34 & .28
\end{tabular}

2. IES-R Intrusion

$\begin{array}{lllll}.45 & .89 & .40 & .39 & .32\end{array}$

3. IES-R Avoidance

$\begin{array}{llll}.43 & .13 & .14 & .08\end{array}$

4. IES-R Hyperarousal

$\begin{array}{lll}.36 & .35 & .31\end{array}$

5. Fear of COVID-19

$.93 \quad .87$

6. Fear of Infection

.66

7. Fear of Death

Note. The table shows rank correlations, i.e. linear correlations on rank data. Correlations of .20 and larger had $B F_{10}>3$, whereas correlations of .24 and larger had $B F_{10}>10$. Correlations of .16 and larger had uncorrected $p$-values less than .05, whereas correlations of .26 and larger had uncorrected $p$-values less than .001 . 
Running head: PSYCHOLOGICAL IMPACT OF COVID-19 IN PREGNANCY

Table 4

Correlates of IES-R and COVID-19 Fear Scales

\begin{tabular}{|c|c|c|c|c|c|c|c|}
\hline \multirow[b]{2}{*}{ Variable } & \multicolumn{4}{|c|}{ IES-R } & \multicolumn{3}{|c|}{ COVID-19 Fear } \\
\hline & Total & Intrusion & Avoidance & Hyperarousal & Total & Infection & Death \\
\hline Support Total & -.03 & -.06 & .11 & -.09 & .03 & .01 & .06 \\
\hline Support Partner & $-.21 *$ & $-.20 *$ & -.07 & $-.26^{*}$ & $-.22 *$ & $-.25 *$ & -.16 \\
\hline Support Mother & .12 & .07 & $.21 *$ & .03 & .12 & .09 & .12 \\
\hline Support Others & -.08 & -.11 & .08 & -.11 & .12 & .08 & .14 \\
\hline RQ Secure & $-.20 *$ & $-.19 *$ & -.03 & $-.29 *$ & -.04 & -.03 & -.05 \\
\hline RQ Fearful & $.18^{*}$ & $.18^{*}$ & .01 & $.19 *$ & .04 & .03 & .07 \\
\hline RQ Preoccupied & .15 & $.19^{*}$ & .04 & $.21^{*}$ & .01 & .05 & -.01 \\
\hline RQ Dismissing & -.02 & .00 & -.01 & .05 & -.06 & -.03 & -.05 \\
\hline Age & .05 & .10 & -.02 & .06 & .02 & .02 & .00 \\
\hline Gestation week & .07 & .07 & .03 & .05 & .06 & .08 & .03 \\
\hline Financial status & -.15 & -.11 & $-.25 *$ & -.07 & $-.26^{*}$ & $-.28 *$ & $-.20 *$ \\
\hline Educational level & .03 & .03 & .04 & -.05 & -.07 & -.10 & -.02 \\
\hline
\end{tabular}


Figure 1. Network model of relations among significant correlates of psychological reactions to the COVID-19 epidemic among pregnant women. Solid lines denote positive correlations, whereas dashed lines denote negative correlations. The thickness of lines indicates the size of correlations. Correlations less than .10 are suppressed.

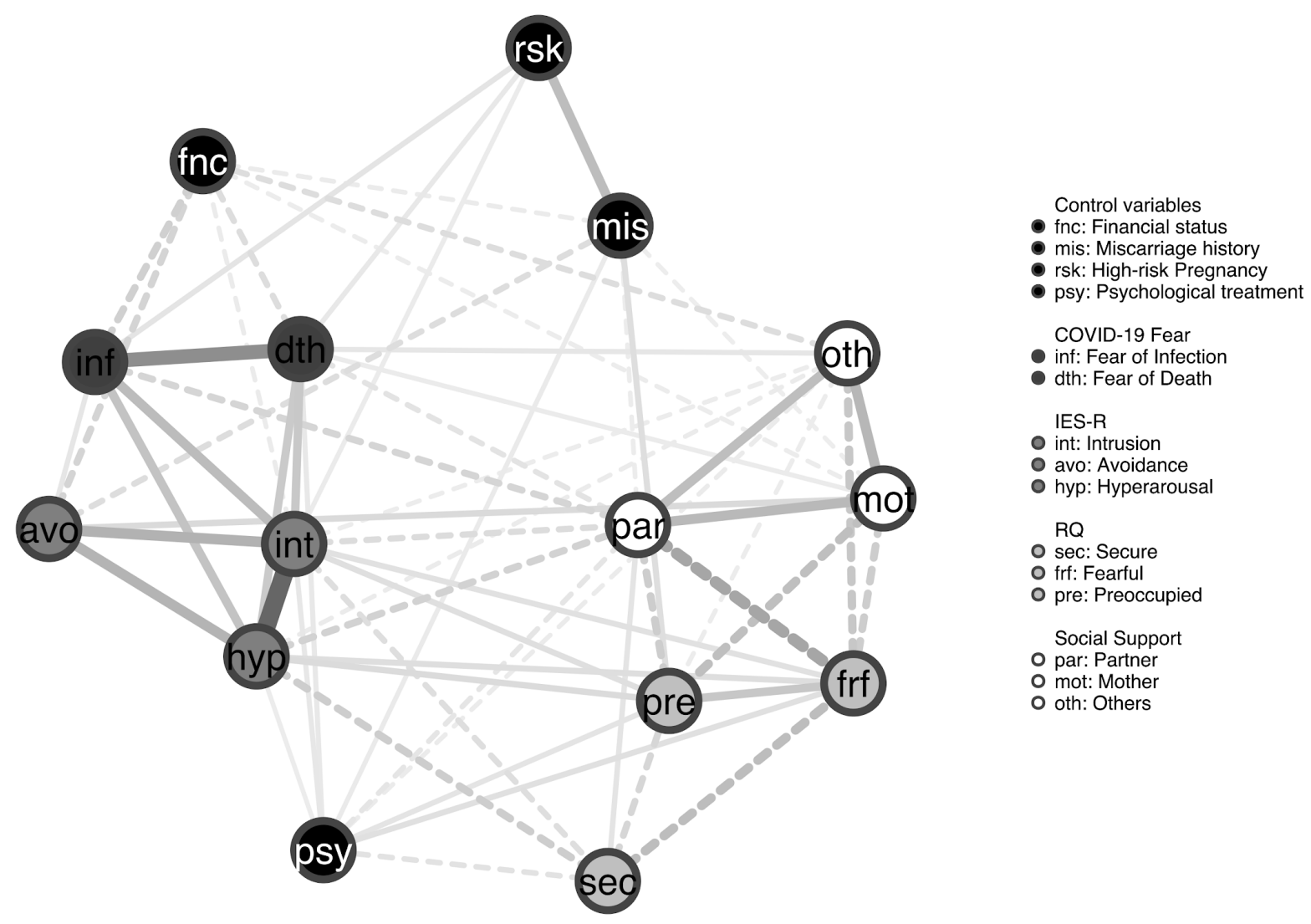

